\title{
HPLC Detectors, Their Types and Use: A Review
}

\author{
Ashrani Sunil ${ }^{1 *}$, Goyal Anju ${ }^{2}$ and Vaishnav Rajat ${ }^{2}$ \\ ${ }^{1}$ Department of M Pharma Research Scholar, Pharmaceutical Quality Assurance, Bhupal Nobles' University, India \\ ${ }^{2}$ Department of Pharmaceutical Quality Assurance, Professor Bhupal Nobles' University, India
}

Submission: May 07, 2018; Published: May 29, 2018

"Corresponding author: Sunil Ashrani, M Pharma Research ScholarBhupal Nobles' University, Udaipur, India, Tel: 7425839345;

Email: sunilashrani93@gmail.com

\begin{abstract}
HPLC is one of the most powerful techniques for separation and quantitative determination of the constituents in a mixture. It works on the principle of Affinity chromatography having two phases viz: stationary and mobile phase. The constituent with lower affinity for stationary phase travels faster and vice-versa. A detector in HPLC is placed at the end of the system. Its work is to analyse the solution which is eluting from the column. Detectors are broadly classified into two groups: Bulk property detectors and solute property detectors. Bulk property detectors (Nonselective detectors) are those that measure the changes in solute and mobile phase in combination. Such detectors show fluctuation in readings even with slight change in mobile phase combination. Solute property detectors are also called as selective detectors because they give response for a particular physical or chemical property of the analyte, being ideally independent of the mobile phase. Other than that there are numerous newer developments made in detectors for more accurate determination. The present paper is meant on to focus on different detectors used in HPLC, there area of utilisation and the advancement made in recent time.
\end{abstract}

Keywords: HPLC; Detectors; Bulk Property; Solute Property; Chromatography

\section{Introduction}

The most powerful technique to determine quantitatively and separate the mixture of composition in today's modern chemistry is Chromatography especially High Performance Liquid Chromatography or High Pressure Liquid Chromatography [1]. HPLC works on the principle of Affinity chromatography. The solution of the sample is injected into a column of a porous material (stationary phase) and a liquid (mobile phase) is pumped at high pressure through the column. The mixture on travelling through the stationary phase splits into its constituents and the component with high affinity for stationary phase travels late whereas one with less affinity elutes fast. This is also based partition coefficient of the material [2].

\section{Instrumentation of HPLC (Figure 1)}

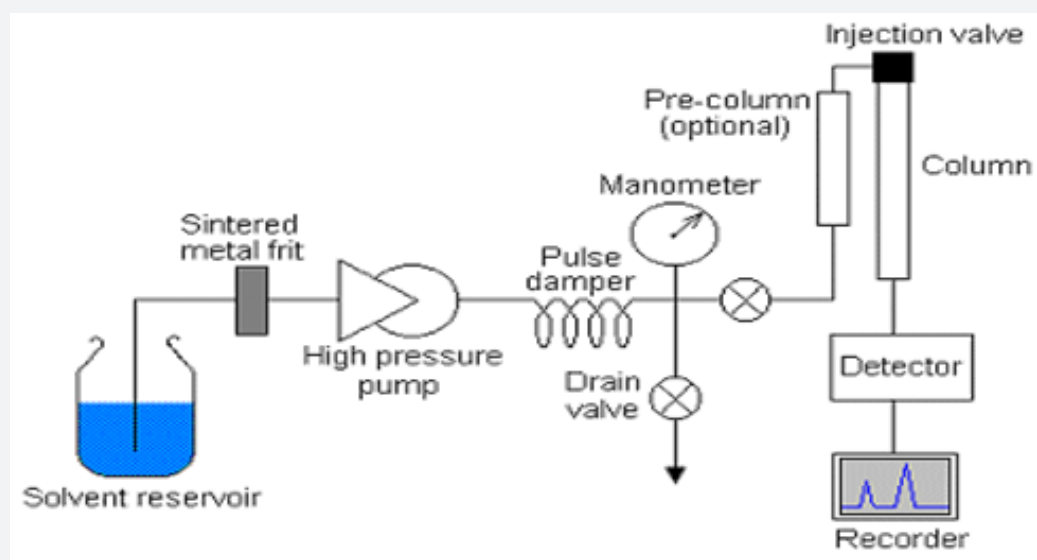

Figure 1: Schematic representation of HPLC system [3]. 


\section{Detectors}

A detector in HPLC is placed at the end of the system. Its work is to analyse the solution which is eluting from the column. The concentration of individual component of the analyte is proportional to the electronic signal coming out of the component of the mixture $[3,4]$.

\section{Features of Detectors Used In HPLC}

a) It must show response for all the components in the mixture.

b) It must show a linear response to the concentration of the analyte.

c) Temperature variation must not affect the response.

d) It must be independent of eluent composition (gradient).

e) It must be capable of tracing even lower concentrations.

f) The peaks must not be widened.

g) It must produce stable and reproducible signal.

h) It should be non destructive [5].

\section{Types of Detectors}

Detectors are broadly classified into two groups:

Bulk Property Detectors: Bulk property detectors are those that measure the changes in solute and mobile phase in combination. Such detectors show fluctuation in readings even with slight change in mobile phase combination. Examples are: refractive index and conductivity detectors. Due to poor sensitivity and limited range, they are used less despite being universally applicable. In general they are called as non selective detectors because they react to the bulk property of the analyte.

Solute Property Detectors: Solute property detectors are also called as selective detectors because they give response for a particular physical or chemical property of the analyte, being ideally independent of the mobile phase. Practically, it is not possible to achieve complete independence from mobile phase but the signal discrimination usually makes it sufficient to work with solvent changes as in gradient elution $[6,7]$.

\section{Bulk Property Detectors}

Electrical Conductivity HPLC Detectors: These detectors senses all the ions, whether they are from a solute, or from the mobile phase. It measures the conductivity of mobile phase along with the solute which needs to be backed-off by suitable electronic adjustments. Thus it is a type of Electrical Conductivity Detector. The measured electronic resistance is directly proportional to the concentration of ions present in the solution [8].
Refractive Index HPLC detectors: They are also one of the bulk property detectors and are based on the change of the refractive index of the eluent from the column with respect to pure mobile phase [9]. There aredifferent types of Refractive index detectors: Christiansen effect detector, interferometer detector, thermal lens detector and the dielectric constant detector. They are mostly used for detection of non-ionic compounds that neither fluoresce nor absorb in the UV region. They face the drawback of being less sensitive, need of temperature control and less suitability to gradient elution [10].

Electrochemical HPLC Detectors: They are termed as "Electrochemical detectors" for the reason being that they usually measure the current associated with the oxidation or reduction of solutes. They act as amperometer or coulometer in HPLC [11]. They are classified as equilibrium and dynamic detectors. The suitability of these detectors depends on the volumetric characteristics of the solute molecules in the aqueous or organic mobile phase. They are sensitive to changes in the flow rate or composition of the eluent and require working electrode, reference electrode and auxiliary electrode [12].

Light Scattering HPLC Detectors: Light scattering HPLC detectors are useful for large molecular weight molecules like surfactants, lipids and sugar. It measures the scattered light coming out of the eluent. Low angle laser light scattering detector and the multiple angle laser light scattering detectors are the two types of Light scattering detectors available. They are also called as Evaporative light scattering detector because in this the beam of light by particles of compound remaining after evaporation of the mobile phase. The importance of such type of detector is growing with time because it acts as universal detector and does not require a compound to have a chromophore for detection. They can be used with gradient elution [13].

\section{Solute Property Detectors}

Ultraviolet/Visible Detectors: The most common HPLC detectors used are UV detectors because of the fact that most of the compounds absorb in UV or visible region. They give specific response to the class of compounds or particular compounds depending upon the functional group of eluting molecules [14]. The basis of working for optical detectors is the change in intensity when a beam of electromagnetic radiation passes through the detector flow cell. These detectors are of three different types: Fixed wave length detectors, variable wavelength detectors and diode array detectors.

Fixed Wave Length Detectors: Such type of detectors does not allow change in wavelength of the radiation. They are difficult to find nowadays due to limitation of working wavelength. Low pressure mercury lamp is used for very intense light at $253.7 \mathrm{~nm}$ or $254 \mathrm{~nm}$. 
Variable Wavelength Detectors: Variable wavelength detectors can be adjusted to work on any wavelength over full UV- visible region. The wavelength can be selected at $3 \mathrm{~nm}$ or less.

Diode Array Detectors: In diode array detector, the sample is subjected to light of all wavelengths generated by the lamp at once. The lights from emission source when collimated by an achromatic lens system, the total light passes through the detector cell onto a holographic grating and then falls onto a diode array which contains 100 s of diodes. The chromatogram can be produced using the UV wavelength that was falling on that particular diode at the end of the run. DAD helps to see the response of the analyte at different wavelengths in only single run and thus saves time and energy [15].

\section{Fluorescence HPLC Detectors}

Fluorescent detectors are the most selective, sensitive and specific than all other HPLC detectors. Specific wavelength is used to excite and then emit light signal in analyte atoms. They intensity of light is monitored continuously to quantify the analyte concentration. Fluorescence is $10-1000$ times sensitive than UV detector for strong UV absorbing compounds. Even a single analyte in the cell can be detected by the fluorescence detector. For some compounds which do not have fluorescence absorbance or low absorbance, they can be treated with fluorescence derivatives such as dansylchloride [16]. The system is easy to operate and relatively stable. There are three types of Fluorescence detector:

Single Wavelength Excitation Fluorescence Detector: The wavelength of excitation is fixed in such type of detectors and they are made from low pressure mercury lamp.

Multi Wavelength Fluorescence Detector: It contains two monochromators to select wavelength of excitation.

Laser Induced Fluorescence Detector (LIFDs): It shows optical emission from molecules that have excited to higher energy levels by absorption of electromagnetic radiations. It is used as separating tool for polymerase chain reactions, determination of solutes like proteins, nucleic acids, polycyclic aromatic hydrocarbons and toxic elements like cyanide [17].

Mass Spectrometric HPLC Detectors: The detection by mass spectrometers is based on molecular fragmentation by electric fields and separation is based on the mass to charge ration of the fragmented molecule. They show high selectivity and sensitivity and are used in LC-MS technique for advantage in terms of resolution and sensitivity. The analyte for MSD must be in ionic form.

\section{Properties of Mass spectrometric detectors}

a) Co-eluting peaks which are not separated chromatographically can be isolated and separated on the basis of their mass. b) The molecular mass of unknown molecules can be known.

c) It is fast detection method without the need of retention time validation.

d) It is quantitative as well as qualitative analysis [18].

\section{Infrared Detector}

Infrared detectors are chosen on the basis of property of compound to absorb infrared light i.e. it falls in the region of 4000-690 cm-1. It is a sophisticated instrument and requires Cells or windows made of $\mathrm{NaCl}$ or $\mathrm{CaF} 2$ and the wavelength scanning is provided by semicircular filter wedges. The detector is suitable for polymer analysis as it differentiate the secondary structure.

\section{The Detector Holds Some Limitations}

A. The mobile phase must be chosen cautiously so that it does not absorb in the region of the analyte.

B. The buffers used must be volatile in nature [19].

\section{Other Popular Detectors Used In HPLC:}

a) Transport Detectors: The transport detectors have a carrier like metal chain, wire or disc which passes continually through column eluting the analyte of interest from the mobile phase. The solute is adhered on the surface as thin film whereas the mobile phase evaporates. Moving wire and Chain detectors are the two types of transport detectors available for HPLC system.

b) Chiral Detectors: There are compounds and drugs that exist in their enantiomeric form but may have different pharmacological properties. The separation of these enantiomers is possible by the detectors capable of responding to the different chiral forms. They are used for the optically active compounds like amino acids, terpenes and sugars and other compounds. Polarimetry or Optical rotatory dispersion (ORD) and Circular dichroism (CD) are two techniques of chiral detection. ORD detectors are based on differences in refractive index whereas CD detectors differentiate enantiomers by measuring differences between the absorption of right and left handed circularly polarised lights.

c) Corona Discharge Detectors: Corona charged aerosol detection (CAD), sometimes referred to as corona discharge detection (CDD) is a newer and unique technique. In this technique, the HPLC column eluent is first nebulized with a nitrogen (or air) carrier gas to form droplets which are then dried to remove mobile phase, producing analyte particles. The principal of working of Corona discharge detectors is the charge transfer diffusionally to the opposing stream of analyte particles when primary stream of analyte particles is met by a secondary stream of nitrogen (or air). The stream 
of nitrogen is positively charged and produces required high -voltage in the platinum corona wire for diffusion to take place. The analytes then are further transferred to a collector where it is measured by a highly sensitive electrometer, generating a signal in direct proportion to the quantity of analyte present $[20,21]$.

\section{Conclusion}

The work of detector is to detect and give the information to the recorder which shows it in a form of a chromatogram. Every compounds has its own properties which is not completely the same with one another, thus this arises a need to have different detectors for different compounds. Before beginning the separation by HPLC it is thus very important to study about the nature of the compound and select the detector accordingly. The selection of wrong detector misguides our journey of separation and quantification. The paper summarizes different types of detectors used in HPLC with their scope of utilisation in Separation and determination of the compound.

\section{References}

1. Prathap B, Dey A, Srinivasa rao GH, Johnson P, Arthanariswaran P, et al. (2003) A Review-Importance of RP-HPLC in Analytical Method Development. IJNTPS 3(1): 23.

2. Gupta V, Jain ADK, Gill NS, Gupta K (2012) Development and validation of HPLC method-a review. Int Res J Pharm App Sci 2(4): 17-25.

3. Bhardwaj SK, Dwivedi K, Agarwal DD (2015) A Review: HPLC Method Development and Validation. International Journal of Analytical and Bioanalytical Chemistry 5(4): 76-81.

4. Sharma BK (2006) Instrumental methods of chemical analysis. $\left(25^{\text {th }}\right.$ edn.); Goel publishing house, pp. 286-385.

5. Kamboi PC (2010) Pharmaceutical analysis instrumental methods. ( $1^{\text {st }}$ edn.); vallabh publication, Delhi, India, pp:257-265.

6. Skoog DA, Holler FJ, Nieman TA (2004) Principle of Instrumental Analysis. ( $5^{\text {th }}$ edn.); Thomson Book Cole, Singapore, pp. 725-766.
7. Willard HH, Merrit LL, Dean JA, Settle FA (1986) Instrumental methods of analysis. ( $1^{\text {st }}$ edn.); CBS publishers and distributors, pp. 580-613.

8. www.pharmaceuticalguidelines.com

9. Raymond S (1995) Chromatographic detectors design: Function and Operation. Chromatographic Science Series 73: 201-204.

10. Kenmore CK, Ersline SR (1997) Refractive Index Detection by Interferometric Backscatter in Packed-Capillary High Performance Liquid Chromatography. J Chr 762(1-2): 219-225.

11. Willard HH, Dean AJ (1986). Instrumental Methods of Analysis. ( $7^{\text {th }}$ edn.); CBS Publishers and distributors, pp. 513-604.

12. Ismail H, Kassandra K, Dernik G (2005) Electrochemical Imaging of Fusion Pore Openings by Electrochemical Detectors Arrays. Centre of scientific studies 102: 13879-13884.

13. Wang D, Abate D (2010) HPLC: Past and Present. Angew Chem Int 49: $1300-2312$

14. Beckett AH, Stenlake JB (2007) Practical Pharmaceutical Chemistry, Part 2. (1t edn.); CBS publishers and distributors, pp. 157-166.

15. Fourkaridis GN, Muntingh GL, Osuch E (1994) Application of Diode Array Detection for the Identification of Poisoning by Traditional medicines. J Ethnopharmacology 41(3): 135-146.

16. Chatwal GR, Anand SK (2007) Instrumental methods of Chemical analysis. ( $5^{\text {th }}$ edn); Himalaya publishing house, pp. 2624-2639.

17. Details-on-various-detectors-used-in-high-performance-liquidchromatography-in-compare-to-sensitivity

18. Watson DG Pharmaceutical analysis ( $2^{\text {nd }}$ edn.); Elsevier pp. 281-286.

19. Scott, RPW (1996) Chromatographic Detectors. Design, Function and Operation, Marcel Dekker: New York, USA.

20. Michael Swartz (2010) HPLC Detectors: A Brief Review. Journal of Liquid Chromatography \& Related Technologies 33: 1130-1150.

21. Ramni K, Kaur N, Upadhyay A, Suri OP, Thakar A (2011) High Performance Liquid Chromatography Detectors- A Review. IRJP 2(5): 1-7.

\section{Your next submission with Juniper Publishers will reach you the below assets}

- Quality Editorial service

- Swift Peer Review

- Reprints availability

- E-prints Service

- Manuscript Podcast for convenient understanding

- Global attainment for your research

- Manuscript accessibility in different formats

( Pdf, E-pub, Full Text, Audio)

- Unceasing customer service

Track the below URL for one-step submission https://juniperpublishers.com/online-submission.php 